\title{
Decreased serum uric acid in patients with traumatic brain injury or after cerebral tumor surgery
}

Miao He, MD, Jianqiao Zheng, MD, PhD, Hao Liu, MD, PhD, Ying Wu, MD, PhD, Xinsheng Xue, MD, PhD, Chaoran Wu, MD, $P h D$, Huiping $L i, M D, P h D$.

\begin{abstract}

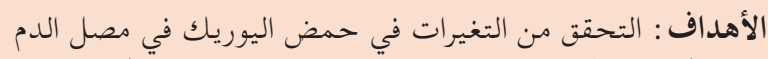

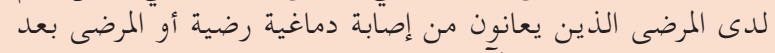

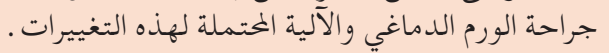

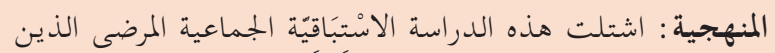

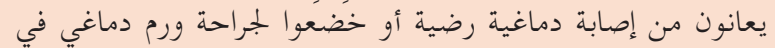

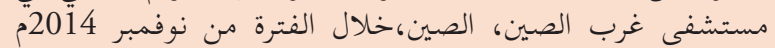

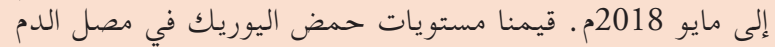

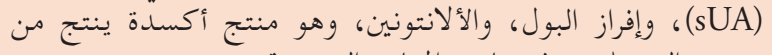
حمض اليوريك، وغيرها من المعايير السريرية.

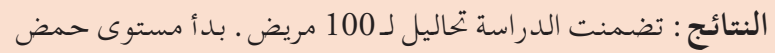

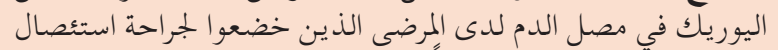

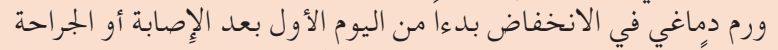

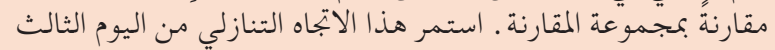

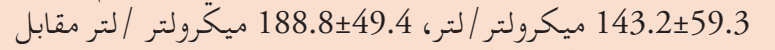

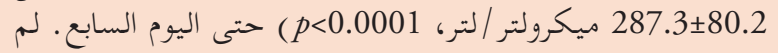

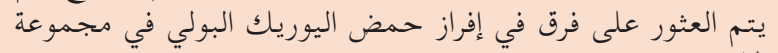

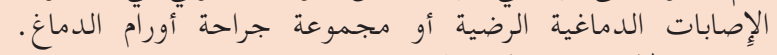

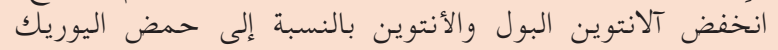

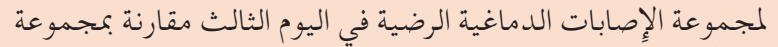

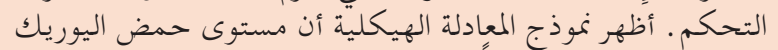

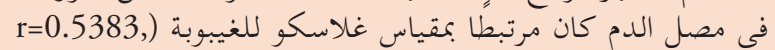

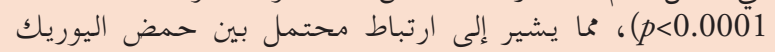

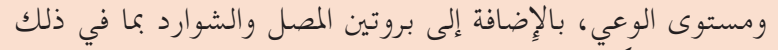

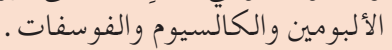

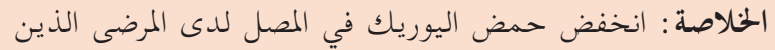

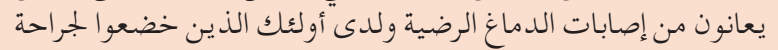

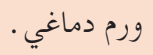

Objectives: To investigate changes in sUA in patients with TBI or patients after cerebral tumor surgery and the possible mechanism of these changes.

Methods: This prospective cohort study enrolled patients with TBI or underwent cerebral tumor surgery at West China Hospital, China, from November 2014 to May 2018. Serum UA (sUA) levels, urine excretion, UA oxidant product allantoin and other clinical parameters were assessed.

Results: 100 patients were enrolled for analysis. sUA in patients with TBI or underwent cerebral tumor surgery started to decline from day 1 after injury or surgery compared to control. This decreasing trend continued from day $3(143.2 \pm 59.3 \mu \mathrm{mol} / \mathrm{L}, 188.8 \pm 49.4 \mu \mathrm{mol} / \mathrm{L}$ vs $287.3 \pm 80.2 \mu \mathrm{mol} / \mathrm{L}, p<0.0001)$ until day 7 . No difference in urinary UA excretion was found in the TBI group or cerebral tumor surgery group. Urine allantoin and the allantoin to sUA ratio of the TBI group decreased on day 3 compared with the control group. The structural equation model showed that the sUA level was related to the Glasgow coma score (GCS) ( $r=0.5383, p<0.0001)$, suggesting the potential association of UA with consciousness level, as well as serum protein and electrolytes including albumin, calcium and phosphate.

Conclusion: The sUA was decreased in patients with TBI or underwent cerebral tumor surgery.

Neurosciences 2021; Vol. 26 (1): 36-44 doi: $10.17712 / n s j .2021 .1 .20200089$

From the Department of Anesthesiology (He, Zheng, Wu), Department of Neurosurgery (Liu), Department of Neurology (Wu), Department of Critical Care Medicine (Xue), West China Hospital of Sichuan University, Sichuan, China

Received 5th June 2020. Accepted 8th August 2020.

Address correspondence and reprint request to: Dr. Huiping Li, Department of Critical Care Medicine, West China Hospital of Sichuan University, Sichuan, China

ORCID ID: https://orcid.org/0000-0002-8691-5740

E-mail: huipingli2154@hotmail.com 
Units ric acid (UA) results from purine metabolism in hominoids. High serum uric acid (sUA), or hyperuricemia, is correlated with gout and many other diseases, including diabetes, hyperlipidemia, kidney diseases and cardiovascular diseases. ${ }^{1}$ Despite the adverse effects of high UA, studies have found that UA has strong endogenous antioxidant effects that protect against oxidative stress. The antioxidant action of UA was supported by evidence from neurodegenerative diseases, such as amyloidosis, Parkinson's disease, and Alzheimer's disease resulting from excessive oxidative damage. ${ }^{2-4}$

Recently, new evidence on UA alterations in acute neuronal injury has emerged. It was found that administration of urate could reduce striatal or cortical damage and preserve neurological function in the context of cerebral ischemia., Although the precursor of UA, inosine, has been found to produce a better recovery from traumatic brain injury (TBI) in animal models with similar metabolic injury, the role of uric acid remains unclear. Some clinical studies on severe TBI as well as subarachnoid hemorrhage ( $\mathrm{SAH}$ ) patients identified decreased sUA in these patients, ${ }^{7,8}$ while other research mentioned increased sUA in patients with TBI. ${ }^{9}$ In patients with cerebral tumors, surgery may also trigger inflammatory and oxidative injuries resulting in neurological dysfunction. Whether UA levels change in patients undergoing cerebral tumor surgery is unclear.

This study investigated changes in sUA in patients with TBI or patients after cerebral tumor surgery and the possible mechanism of these changes.

Methods. The prior related researches were searched in Web of Science, Pubmed, Ovid Medline and Google Scholar. This prospective cohort study was approved by the Ethics Committee of West China Hospital of Sichuan University. Patients admitted to the Intensive Care Unit (ICU) in West China Hospital due to TBI or to undergo cerebral tumor surgery from November 2014 to July 2018 were enrolled. Patients received into the Department of Neurology due to peripheral neurological diseases were recruited as the control group. The inclusion, exclusion and withdrawal criteria were as follows.

Disclosure. This study was funded by National Natural Science Foundation of China (81100189 and 81471141).
Inclusion criteria: (1) patients who agreed to be involved in the study and sign the informed consent form; (2) patients with TBI or patients who underwent cerebral tumor surgery and were admitted to the ICU; patients with peripheral neurological disease; (3) patients with an estimated ICU or hospital stay (for the control group) of longer than 7 days; (4) patients aged from 18-75 years.

Exclusion criteria: (1) history of hyperuricemia (sUA >420 $\mu \mathrm{mol} / \mathrm{l})$; (2) history of gout, diabetes mellitus, or renal diseases; amyloidosis, Parkinson's disease, Alzheimer's disease; or other neurodegenerative diseases; (3) history of glaucoma or multiple sclerosis; (4) obesity; pregnancy; and (5) acute central nervous system viral infections or stroke.

Withdrawal criteria: (1) life-threatening complications during the ICU or hospital stay; (2) acute kidney injury (AKI) during the ICU stay; and (3) failure to obtain samples for any reason.

A planned sample size was 20 per group calculated using the generalized estimating equation (GEE) test with a two-sided 5\% significance level, a timeaveraged difference (TAD) of $110 \mu \mathrm{mol} / \mathrm{L}$, and a standard deviation of $87 \mu \mathrm{mol} / \mathrm{L}^{10}$ and assuming a $10 \%$ drop-out rate. Overall, 113 patients were enrolled in this research. All the participates were given enteral nutrition. The cerebral tumor surgery group and the control group were fed orally, while patients with TBI were fed through nasogastric tubes. Urine and blood samples were collected in the morning on day 1 , day 3 and day 7 after admission after 12 hours of fasting in patients with TBI and the control group. For patients with a cerebral tumor, we collected samples on the first day after admission to the hospital and on days 1,3 , and 7 after surgery. The following parameters were assessed: sUA and 24-hour urine UA (UUA); liver function - aspartate transaminase (AST) and alanine transaminase (ALT); serum total protein (TP), albumin (ALB), and globulin (GLB); fasting blood glucose (Glu); total bilirubin (TBIL), and triglycerides (TGs); cholesterol (Cho); serum creatinine (Cr), serum cysteine (Cys), blood urea nitrogen (BUN), and serum electrolyte levels including those of serum sodium $\left(\mathrm{Na}^{+}\right)$, serum potassium $\left(\mathrm{K}^{+}\right)$, serum calcium $\left(\mathrm{Ca}^{2+}\right)$, serum magnesium $\left(\mathrm{Mg}^{2+}\right)$, serum chlorine $\left(\mathrm{Cl}^{-}\right)$, serum bicarbonate $\left(\mathrm{HCO}_{3}^{-}\right)$, and serum phosphate (Phos); urine $\mathrm{pH}(\mathrm{UpH})$; specific gravity of urine (SPGR); 24-hour urine sodium $\left(\mathrm{UNa}^{+}\right)$, 24-hour urine potassium $\left(\mathrm{UK}^{+}\right)$, and 24-hour urine chlorine $\left(\mathrm{UCl}^{-}\right)$; 24-hour creatinine (UCr); and serum norepinephrine (NE), serum epinephrine (E), 24-hour urine norepinephrine (UNE), and 24-hour urine epinephrine (UE). All urine samples were collected after 24 hours and mixed well 

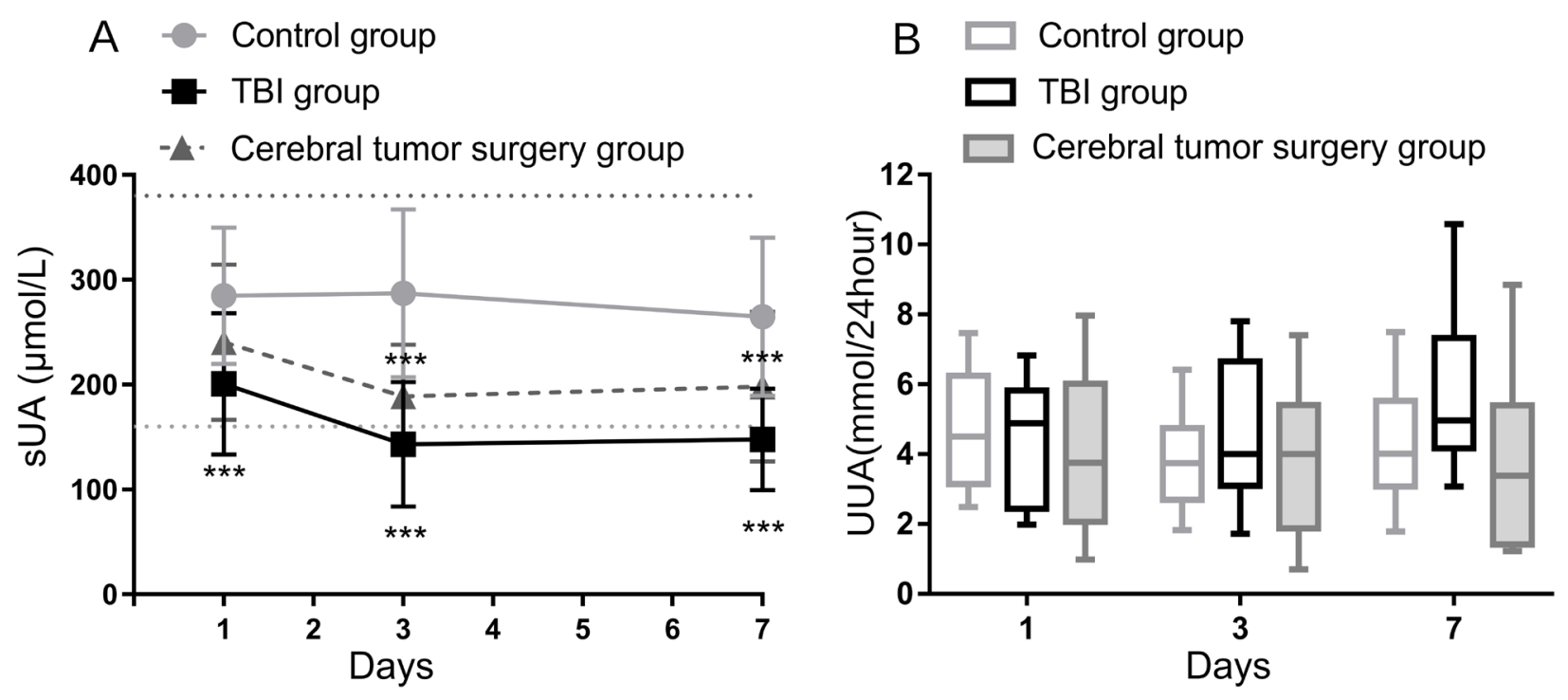

Figure 1 - Change in serum uric acid (sUA) level and 24-hour urine uric acid level (UUA). A) sUA decreased after traumatic brain injury or cerebral tumor surgery. The TBI group and cerebral tumor surgery group were compared with the control group respectively. Dashed lines are the normal range of sUA. ${ }^{* * *} p<0.001$. B) No significant change was found in the TBI group or cerebral tumor surgery group compared with the control group. TBI - traumatic brain injury
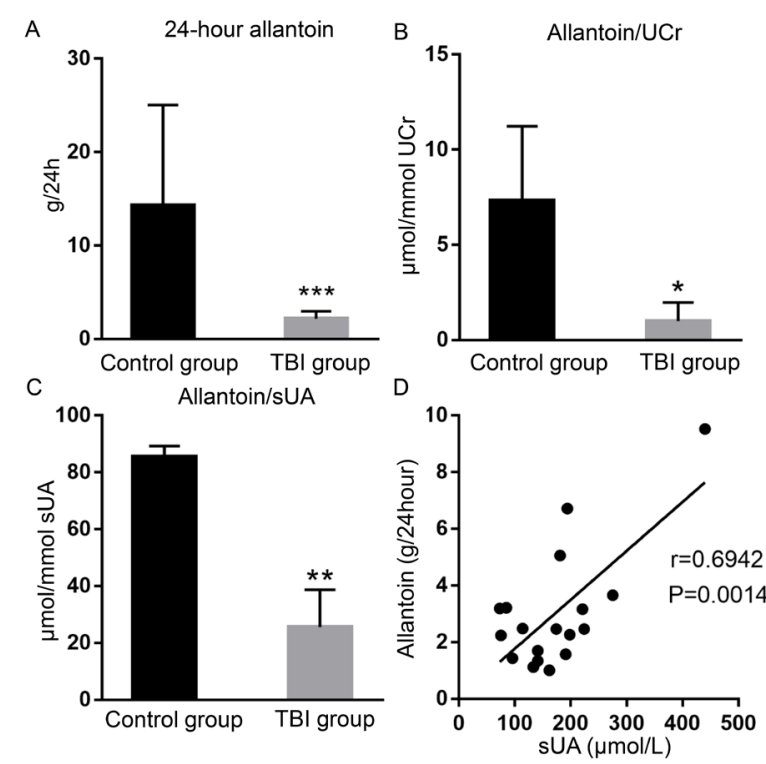

Figure 2 - Change in urine allantoin on day 3 after TBI. A) Allantoin in 24-hour urine. Data are presented as the mean \pm SEM. *** $p=0.0003$. B) Allantoin concentration normalized to urine creatine. Data are presented as the median and quartile. ${ }^{*} p=0.0338$. C) Ratio of allantoin and $s \mathrm{UA}{ }^{* *} p=0.0063$. D) Correlation between allantoin and sUA. TBI - traumatic brain injury. sUA - serum uric acid. UCr - urine creatine before being sent to the lab for testing. Two milliliters of the 24-hour urine samples from the TBI and control groups were collected and stored at $-80^{\circ} \mathrm{C}$ until they were used to assess urine allantoin.

Liquid chromatography tandem mass spectrometry (LC-MS/MS). Allantoin was quantified using LC-MS/ MS as described (Agilent 1200 series HPLC system and Agilent G6460, Agilent Technologies, Santa Clara, CA, USA). ${ }^{11}$ Each $50 \mu \mathrm{l}$ aliquot of urine was added to $50 \mu \mathrm{l} 1 \mu \mathrm{g} / \mathrm{ml}$ Allantoin $-{ }^{13} \mathrm{C}_{2},{ }^{15} \mathrm{~N}_{4}$ (A540502 Toronto Research Chemicals, North York, ON, Canada) and deproteinized with $300 \mu \mathrm{l}$ acetonitrile. After centrifugation at $20000 \times \mathrm{g}$ for $10 \mathrm{~min}, 2 \mu \mathrm{l}$ of supernatant was loaded on a Poroshell 120 Hilic- $_{18}$ column by Agilent $(4.6 \times 100 \mathrm{~mm}, 2.7 \mu \mathrm{m})$. The isocratic mobile phase consisted of $90 \%$ acetonitrile and $10 \%$ water $(0.1 \%$ formic acid $)$ at a column flow rate of 0.3 $\mathrm{ml} \cdot \mathrm{min}^{-1}$. The MS/MS negative-ion mode was used for analysis with multiple reaction monitoring (MRM) acquisition: internal standard (allantoin $-{ }^{13} \mathrm{C}_{2},{ }^{15} \mathrm{~N}_{4}$ ) (at $\mathrm{m} / \mathrm{z} 157.1 / 114$ ) and allantoin (at $\mathrm{m} / \mathrm{z} 163.1 / 100.2$ ). The source parameters were set up as follows: ion source, ESI source; atomizing gas and auxiliary gas, nitrogen; ion source temperature, $350^{\circ} \mathrm{C}$; gas flow rate, $5 \mathrm{~L} / \mathrm{min}$; nebulizer pressure, $45 \mathrm{psi}$; desolvation temperature and sheath gas temperature, $350^{\circ} \mathrm{C}$; sheath gas flow speed, 
Table 1 - Demographics and baselines of patients.

\begin{tabular}{|c|c|c|c|c|}
\hline Variables & $\begin{array}{c}\text { Control group } \\
(\mathrm{n}=49)\end{array}$ & TBI group $(n=32)$ & $\begin{array}{l}\text { Cerebral tumor surgery group } \\
(\mathrm{n}=19)\end{array}$ & $P$-value* \\
\hline Age (years) & $50(10)$ & $47(22)$ & $43(24)$ & 0.0093 \\
\hline Female sex, $\mathrm{n}(\%)$ & $34(69)$ & $12(40)$ & $10(52)$ & 0.0603 \\
\hline BMI $\left(\mathrm{kg} / \mathrm{m}^{2}\right)$ & $23.86 \pm 2.37$ & $22.29 \pm 2.58$ & $23.22 \pm 3.70$ & 0.0931 \\
\hline Diagnosis, $n(\%)$ & & & & NA \\
\hline Peripheral vertigo & $10(20)$ & & & \\
\hline Anxiety or depression status & $21(43)$ & & & \\
\hline Peripheral nerve disease & $6(12)$ & & & \\
\hline Thyroid disorder & $6(12)$ & & & \\
\hline Subarachnoid hemorrhage & & $23(72)$ & & \\
\hline Subdural hemorrhage & & $15(47)$ & & \\
\hline Extradural hemorrhage & & $8(25)$ & & \\
\hline Intracranial hematoma & & $16(50)$ & & \\
\hline Meningioma & & & $5(26)$ & \\
\hline Hypophysoma & & & $7(37)$ & \\
\hline Initial sUA $(\mu \mathrm{mol} / \mathrm{L})$ & $286.8 \pm 65.4$ & $271.7 \pm 81.9$ & & 0.0809 \\
\hline Baseline sUA $(\mu \mathrm{mol} / \mathrm{L})$ & & & $301.9 \pm 49.0$ & \\
\hline ALT (IU/L) & $27.5 \pm 24.4$ & $27.9 \pm 17.6$ & $35.1 \pm 26.1$ & 0.5017 \\
\hline AST (IU/L) $(13-35 \mathrm{IU} / \mathrm{L})^{* *}$ & $22.7 \pm 9.8$ & $41.9 \pm 34.8$ & $26.8 \pm 12.9$ & 0.0014 \\
\hline $\mathrm{TP}(\mathrm{g} / \mathrm{L})(65-85 \mathrm{~g} / \mathrm{L})^{* *}$ & $65.3 \pm 6.0$ & $61.3 \pm 10.9$ & $68.2 \pm 5.6$ & 0.0159 \\
\hline $\operatorname{ALB}(g / L)$ & $40.9 \pm 3.6$ & $37.7 \pm 6.7$ & $42.8 \pm 2.1$ & 0.0013 \\
\hline GLB (g/L) & $24.3 \pm 3.7$ & $23.6 \pm 5.5$ & $25.4 \pm 5.0$ & 0.4711 \\
\hline $\mathrm{Cr}(\mu \mathrm{mol} / \mathrm{L})$ & $56.2 \pm 14.5$ & $64.5 \pm 14.7$ & $64.9 \pm 20.6$ & 0.0251 \\
\hline Cys (mg/L) & $0.87 \pm 0.20$ & $0.76 \pm 0.23$ & $0.94 \pm 0.21$ & 0.0138 \\
\hline BUN (mmol/L) & $4.80 \pm 1.39$ & $4.81 \pm 1.59$ & $4.76 \pm 1.91$ & 0.9927 \\
\hline TGs (mmol/L) & $2.00 \pm 1.74$ & $1.11 \pm 0.92$ & $1.27 \pm 0.88$ & 0.0185 \\
\hline Cho $(\mathrm{mmol} / \mathrm{L})$ & $4.08 \pm 1.07$ & $3.42 \pm 1.10$ & $4.37 \pm 1.60$ & 0.0202 \\
\hline \multicolumn{5}{|l|}{ Serum electrolyte level } \\
\hline $\mathrm{Na}^{+}(\mathrm{mmol} / \mathrm{L})$ & $140.9 \pm 2.1$ & $140.4 \pm 5.4$ & $140.8 \pm 2.8$ & 0.8637 \\
\hline $\mathrm{K}^{+}(\mathrm{mmol} / \mathrm{L})$ & $4.30 \pm 0.30$ & $3.75 \pm 0.47$ & $4.09 \pm 0.41$ & $<0.0001$ \\
\hline $\mathrm{Ca}^{2+}(\mathrm{mmol} / \mathrm{L})$ & $2.21 \pm 0.11$ & $2.06 \pm 0.15$ & $2.28 \pm 0.06$ & $<0.0001$ \\
\hline $\mathrm{Mg}^{2+}(\mathrm{mmol} / \mathrm{L})$ & $0.85 \pm 0.07$ & $0.76 \pm 0.10$ & $0.83 \pm 0.06$ & $<0.0001$ \\
\hline $\mathrm{Cl}^{-}(\mathrm{mmol} / \mathrm{L})$ & $102.9 \pm 2.7$ & $104.1 \pm 9.1$ & $101.7 \pm 3.7$ & 0.3320 \\
\hline $\mathrm{HCO}_{3}^{-}(\mathrm{mmol} / \mathrm{L})$ & $24.2 \pm 3.0$ & $19.4 \pm 3.0$ & $24.4 \pm 1.9$ & $<0.0001$ \\
\hline Phos (mmol/L) & $1.11 \pm 0.19$ & $0.83 \pm 0.22$ & $1.15 \pm 0.18$ & $<0.0001$ \\
\hline \multicolumn{5}{|c|}{$\begin{array}{l}\text { Categorical data are } \mathrm{n}(\%) \text { and continuous data are mean } \pm \mathrm{SD} \text {, median }(\mathrm{IQR}) . \mathrm{NA} \text { - not applicable. }{ }^{*} P \text {-value for patients } \\
\text { between three groups; }{ }^{* *} \text { Reference ranges were showing when the subject demographics showing abnormal. BMI - body } \\
\text { mass index; ALT - alanine transaminase; AST - aspartate transaminase; TP - serum total protein; ALB - albumin; GLB - } \\
\text { globulin; } \mathrm{Cr} \text { - serum creatinine; Cys - serum cysteine; BUN - blood urea nitrogen; TGs - triglycerides; Cho - cholesterol; } \\
\mathrm{Na}^{+} \text {- serum sodium concentrations; } \mathrm{K}^{+} \text {- serum potassium concentrations; } \mathrm{Cl}^{-} \text {- serum chlorine concentrations; } \mathrm{HCO}_{3}^{-}- \\
\text {serum bicarbonate radical; } \mathrm{Ca}^{2+} \text { - serum calcium; } \mathrm{Mg}^{2+} \text { - serum magnesium; Phos - serum phosphate radical }\end{array}$} \\
\hline
\end{tabular}

$11 \mathrm{~L} / \mathrm{min}$; capillary voltage, $3500 \mathrm{~V}$; and auxiliary voltage, $500 \mathrm{~V}$.

Statistical analysis. The statistical analysis was conducted with SAS (version 9.4) for Student's t-test, analysis of variance and Wilcoxon rank sum test. Mplus (version 7.4) was used for structural equation model measurement and model fitting. Descriptive statistics and analysis of variance or Wilcoxon rank sum test, chi-square test, as well as repeated measurement analysis of variance were calculated with data from baseline and day 1, day 3 and day 7 after hospitalization, injury or surgery. Data are presented as the mean $\pm S D$ or median (IQR). Pearson or Spearman correlation coefficients between UA and variables on day 3 were calculated to explore the possible associations. All $p$-values were 2-sided, and $p<0.05$ was used for statistical significance. Results. Change in sUA and UUA. In total, 113 patients were recruited into the study, including 
Table 2 - Measurement of parameters on day 3

\begin{tabular}{|c|c|c|c|c|}
\hline Variables & Control group & TBI group & Cerebral tumor surgery group & ${ }^{*} P$-value \\
\hline GCS & $15(0)$ & $9.5(7)$ & $15(0)$ & $<0.0001$ \\
\hline ALT (IU/L) & $30.8 \pm 32.7$ & $33.3 \pm 22.6$ & $24.3 \pm 18.6$ & 0.1435 \\
\hline AST (IU/L) & $23.7 \pm 15.8$ & $48.7 \pm 31.3$ & $19.7 \pm 15.9$ & $<0.0001$ \\
\hline $\mathrm{TP}(\mathrm{g} / \mathrm{L})$ & $63.4 \pm 5.1$ & $54.2 \pm 6.3$ & $58.6 \pm 6.2$ & $<0.0001$ \\
\hline $\operatorname{ALB}(g / L)$ & $40.7 \pm 3.8$ & $31.2 \pm 4.4$ & $35.6 \pm 4.8$ & $<0.0001$ \\
\hline GLB (g/L) & $22.6 \pm 3.2$ & $23.0 \pm 3.2$ & $23.0 \pm 2.7$ & 0.9001 \\
\hline TBIL ( $\mu \mathrm{mol} / \mathrm{L})$ & $11.29 \pm 6.30$ & $13.47 \pm 5.79$ & $13.90 \pm 7.07$ & 0.2899 \\
\hline Glu (mmol/L) & $4.61 \pm 0.48$ & $7.55 \pm 2.28$ & $5.83 \pm 0.99$ & $<0.0001$ \\
\hline $\mathrm{Cr}(\mu \mathrm{mol} / \mathrm{L})$ & $60.9 \pm 15.3$ & $62.7 \pm 20.7$ & $58.1 \pm 21.3$ & 0.7311 \\
\hline Cys (mg/L) & $0.89 \pm 0.20$ & $0.78 \pm 0.26$ & $0.88 \pm 0.20$ & 0.1105 \\
\hline $\mathrm{BUN}(\mathrm{mmol} / \mathrm{L})$ & $4.55 \pm 1.64$ & $4.59 \pm 1.97$ & $4.84 \pm 1.51$ & 0.8528 \\
\hline \multicolumn{5}{|l|}{ Lipid metabolism } \\
\hline TGs $(\mathrm{mmol} / \mathrm{L})$ & $1.87 \pm 1.20$ & $1.31 \pm 0.64$ & $0.89 \pm 0.38$ & 0.0014 \\
\hline Cho $(\mathrm{mmol} / \mathrm{L})$ & $4.09 \pm 1.14$ & $3.48 \pm 0.80$ & $4.12 \pm 1.38$ & 0.0583 \\
\hline \multicolumn{5}{|l|}{ Urine parameters } \\
\hline $\mathrm{UpH}$ & $6.07 \pm 0.89$ & $6.80 \pm 0.73$ & $7.27 \pm 0.53$ & 0.0034 \\
\hline SPGR & $1.015 \pm 0.004$ & $1.023 \pm 0.012$ & $1.012 \pm 0.007$ & 0.0053 \\
\hline $\mathrm{UNa}^{+}(\mathrm{mmol} / 24 \mathrm{~h})$ & $268.0 \pm 95.7$ & $291.2 \pm 167.0$ & $325.5 \pm 204.6$ & 0.3874 \\
\hline $\mathrm{UK}^{+}(\mathrm{mmol} / 24 \mathrm{~h})$ & $42.60 \pm 21.88$ & $83.36 \pm 41.83$ & $43.94 \pm 18.58$ & $<0.0001$ \\
\hline $\mathrm{UCl}^{-}(\mathrm{mmol} / 24 \mathrm{~h})$ & $244.7 \pm 104.6$ & $319.0 \pm 160.8$ & $273.6 \pm 171.8$ & 0.1566 \\
\hline \multicolumn{5}{|c|}{ Serum electrolyte level } \\
\hline $\mathrm{Na}^{+}(\mathrm{mmol} / \mathrm{L})$ & $141.2 \pm 2.6$ & $144.5 \pm 8.1$ & $138.7 \pm 4.4$ & 0.0055 \\
\hline $\mathrm{K}^{+}(\mathrm{mmol} / \mathrm{L})$ & $4.11 \pm 0.32$ & $3.96 \pm 0.41$ & $3.83 \pm 0.69$ & 0.1378 \\
\hline $\mathrm{Ca}^{2+}(\mathrm{mmol} / \mathrm{L})$ & $2.19 \pm 0.10$ & $2.01 \pm 0.08$ & $2.12 \pm 0.10$ & $<0.0001$ \\
\hline $\mathrm{Mg}^{2+}(\mathrm{mmol} / \mathrm{L})$ & $0.86 \pm 0.06$ & $0.89 \pm 0.08$ & $0.87 \pm 0.07$ & 0.1810 \\
\hline $\mathrm{Cl}^{-}(\mathrm{mmol} / \mathrm{L})$ & $102.2 \pm 3.6$ & $107.2 \pm 9.3$ & $99.3 \pm 3.9$ & 0.0003 \\
\hline $\mathrm{HCO}_{3}^{-}(\mathrm{mmol} / \mathrm{L})$ & $24.7 \pm 2.5$ & $24.1 \pm 3.6$ & $24.7 \pm 3.1$ & 0.7620 \\
\hline Phos $(\mathrm{mmol} / \mathrm{L})$ & $1.17 \pm 0.19$ & $0.80 \pm 0.22$ & $1.08 \pm 0.22$ & $<0.0001$ \\
\hline \multicolumn{5}{|l|}{ Catecholamine } \\
\hline $\mathrm{NE}(\mathrm{ng} / \mathrm{L})$ & $220(139)$ & $305(275)$ & $72.5(78)$ & $<0.0001$ \\
\hline $\mathrm{E}(\mathrm{ng} / \mathrm{L})$ & $92(53)$ & $77(59)$ & $52.5(57)$ & 0.0251 \\
\hline \multicolumn{5}{|c|}{$\begin{array}{l}\text { Continuous data are mean } \pm \mathrm{SD} \text {, median }(\mathrm{IQR}) .{ }^{*} P \text {-value for patients between three groups. GCS - Glasgow Coma } \\
\text { Scale, ALT - alanine transaminase, AST - aspartate transaminase, TP - serum total protein, ALB - albumin, GLB - } \\
\text { globulin, TBIL - total bilirubin, Glu - fasting blood glucose, } \mathrm{Cr} \text { - serum creatinine, Cys - serum cysteine, BUN - blood } \\
\text { urea nitrogen, } \mathrm{UpH} \text { - } \mathrm{pH} \text { in urine, } \mathrm{SPGR} \text { - specific gravity of urine, } \mathrm{UNa}^{+}-24 \text {-hour urine sodium concentrations, } \\
\mathrm{UK}^{+} \text {- 24-hour urine potassium concentrations, } \mathrm{UCl}^{-}-24 \text {-hour urine chlorine concentrations, TGs - triglycerides, Cho } \\
\text { - cholesterol, } \mathrm{Na}^{+} \text {- serum sodium concentrations, } \mathrm{K}^{+} \text {- serum potassium concentrations, } \mathrm{Ca}^{2+} \text { - serum calcium, } \mathrm{Mg}^{2+} \text { - } \\
\text { serum magnesium, } \mathrm{Cl}-\text { - serum chlorine concentrations, } \mathrm{HCO}_{3}^{-} \text {- serum bicarbonate radical, Phos - serum phosphate } \\
\text { radical, } \mathrm{NE} \mathrm{-} \mathrm{serum} \mathrm{norepinephrine,} \mathrm{E} \text { - serum epinephrine }\end{array}$} \\
\hline
\end{tabular}

41 patients with TBI, 19 patients who underwent cerebral tumor surgery and 53 patients with peripheral neurological diseases as controls. Among these patients, 7 patients were excluded because of hyperuricemia or AKI, and 6 patients were excluded because of incomplete data collection.

Patient baseline characteristics are shown in Table 1. For patients with TBI, all baseline criteria were assessed immediately after admission to the hospital. The baseline characteristics of cerebral tumor surgery patients and the patients in the control group were collected on day 1 after admission to the hospital. There were no significant differences in sex, body mass index (BMI), or age in patients with TBI or tumor surgery patients compared with the same parameters in the control group. Among patients with TBI, the median 
Table 3 - Pearson or Spearman correlation coefficients between UA and other variables.

\begin{tabular}{|c|c|c|}
\hline Variables & Coefficient & $P$-value \\
\hline GCS & 0.5383 & $<0.0001$ \\
\hline \multicolumn{3}{|c|}{ Protein nutrition status } \\
\hline $\mathrm{TP}$ & 0.52546 & $<0.0001$ \\
\hline ALB & 0.58836 & $<0.0001$ \\
\hline Glu & -0.57723 & $<0.0001$ \\
\hline \multicolumn{3}{|c|}{ Renal function } \\
\hline Cys & 0.41582 & 0.0001 \\
\hline $\mathrm{Cr}$ & 0.27199 & 0.0147 \\
\hline $\mathrm{UK}^{+}$ & -0.27282 & 0.0319 \\
\hline \multicolumn{3}{|c|}{ Serum electrolyte level } \\
\hline $\mathrm{Ca}^{2+}$ & 0.61157 & $<0.0001$ \\
\hline Phos & 0.53801 & $<0.0001$ \\
\hline \multicolumn{3}{|c|}{ Catecholamine } \\
\hline $\mathrm{NE}$ & 0.11140 & 0.3658 \\
\hline $\mathrm{E}$ & 0.27420 & 0.0216 \\
\hline \multicolumn{3}{|c|}{$\begin{array}{l}\text { TP - serum total protein; ALB - albumin; Glu - fasting blood } \\
\text { glucose; Cys - serum cysteine; } \mathrm{Cr} \text { - serum creatinine; } \mathrm{UK}^{+}-24- \\
\text { hour urine potassium concentrations; } \mathrm{Ca}^{2+} \text { - serum calcium; } \mathrm{Mg}^{2+} \\
\text { - serum magnesium; Phos - serum phosphate radical; } \mathrm{NE} \mathrm{-} \mathrm{serum} \\
\text { norepinephrine; E - serum epinephrine }\end{array}$} \\
\hline
\end{tabular}

duration before the first blood sample collection was 10(8) hours. ALB, TGs, $\mathrm{Ca}^{2+}$, and Phos decreased in the TBI group, as shown in Table 1 .

The sUA was significantly decreased on day 1 after TBI or cerebral tumor surgery $(200.9 \pm 67.2 \mu \mathrm{mol} / \mathrm{L}$, $240.5 \pm 74.1 \mu \mathrm{mol} / \mathrm{L}$ vs control group $284.9 \pm 65.2$ $\mu \mathrm{mol} / \mathrm{L}, p=0.0003)$ and was below the normal range on day $3(143.2 \pm 59.3 \mu \mathrm{mol} / \mathrm{L}, 188.8 \pm 49.4 \mu \mathrm{mol} / \mathrm{L}$ vs $287.3 \pm 80.2 \mu \mathrm{mol} / \mathrm{L}, p<0.0001$ ) (Figure $1 \mathrm{~A}$ ). The decrease continued through day $7(147.9 \pm 48.4 \mu \mathrm{mol} / \mathrm{L}$, $198.1 \pm 71.5 \mu \mathrm{mol} / \mathrm{L}$ vs $264.9 \pm 75.6 \mu \mathrm{mol} / \mathrm{L}, p<0.0001)$. UUA was also measured to investigate whether increased UA excretion from urine was attributed to changes in sUA, but no significant difference in UUA was detected among the 3 groups ( $p=0.417$ ) (Figure 1B).

Decreased urine allantoin. To further study the possible causes of the decreased UA, urine allantoin was measured, and a significant decrease in 24-hour excretion of allantoin on day 3 was found $(2.150 \pm 0.823$ $\mathrm{g} / 24 \mathrm{~h}$ vs $14.297 \pm 10.730 \mathrm{~g} / 24 \mathrm{~h}, p=0.0003$ ) in patients with TBI compared with that of patients in the control group (Figure 2A). There was also a dramatic difference in patients with TBI compared to the control group in the ratio of excretion of allantoin to UUA $(2.510$ (1.229) $\mathrm{mmol} / \mathrm{mol}$ UUA vs 13.594 (7.986) $\mathrm{mmol} / \mathrm{mol}$ UUA, $p=0.0085$ ), as well as the ratio of the allantoin concentration to sUA (25.608 (13.119) vs 85.542 (3.643) $\mu \mathrm{mol} / \mathrm{mol} \mathrm{UA} p=0.0063$ ) (Figure 2B) after normalization according to UCr $(0.991(0.977) \mathrm{mmol} /$ mol UCr vs $4.637(4.410) \mathrm{mmol} / \mathrm{mol} \mathrm{UCr}, p=0.0338)$ (Figure 2C).

Renal filtration and reabsorption assessment. To further investigate whether a change in sUA was related to renal filtration and reabsorption, serum cystatin, BUN, creatinine and electrolytes in serum and urine were also assessed. No significant changes were found in cystatin, BUN or creatinine (Table 2).

Of the other parameters assessed in the study, several were decreased in both the TBI and cerebral tumor surgery groups, including ALB $(p<0.0001)$, TGs $(p=0.0014), \mathrm{Ca}^{2+}(p<0.0001)$, and Phos $(p<0.001)$, while NE was increased only in the TBI group (Table 2 ).

Association between UA and other variables. To explore the potential mechanism of sUA changes, either Pearson correlation coefficient or Spearman correlation coefficient was used. The results are shown in Table 3: GCS, TP, ALB, Glu, Cys, $\mathrm{Ca}^{2+}$, and Phos had medium correlations with sUA.

To further investigate the possible association between sUA and the above variables, multiple linear regression (MLR) was performed using data from day 3 , because sUA levels were still declining, indicating that the pathological process had not ceased at that time point.

Modified structural equation models (SEM) were created to explain the relationship between sUA and the variables. Based on the MLR results, the structure of the primary model included the following variables: GCS, TP, ALB, Glu, $\mathrm{Ca}^{2+}$, Cys, Phos, UK, and E. After deleting the insignificant variables and paths, the final model fit well with a posterior predictive P-value of 0.250 and a Bayesian information criterion (BIC) of 1561.025. Five variables (GCS, Glu, $\mathrm{Ca}^{2+}$, Phos, ALB) were identified that might have a relationship with the change in sUA, indicating that the severity of brain injury, glucose metabolism, calcium and phosphorus metabolism, and protein status may be related to sUA level.

Discussion. As the end product of purine metabolism in hominoids, sUA levels in humans are significantly higher than those in other mammals due to the loss of uricase activity in the human body. ${ }^{12} \mathrm{UA}$ has a strong endogenous antioxidant effect in some oxidative stress-induced neurodegenerative diseases and is also involved in numerous disorders, including cardiovascular disease, obesity, diabetes, leukoaraiosis, as well as inflammation. ${ }^{2,3,13-16}$ Although UA may exert 
an important function in protecting neurons from inflammatory injuries, it is still not clear what the role of UA is in brain injury patients. Some studies have found that a low GCS was related to a low UA level in patients with TBI, suggesting that UA may have some function in the recovery from consciousness. ${ }^{8}$ It has also been found that administration of urate could reduce striatal or cortical damage and preserve neurological function in patients with cerebral ischemia., ${ }^{5,6}$ Although the precursor of UA inosine improves the outcome of TBI in animal models, changes in sUA and its role in patients with TBI remain controversial. In severe TBI and SAH patients, decreased sUA was found, ${ }^{7,8}$ while another study on TBI patients showed increased sUA ${ }^{9}$ Hence, our study aimed to elucidate the changes in sUA in patients with TBI. Additionally, sUA changes in cerebral tumor surgery patients were also assessed.

The UA pool in the human body is formed from the dietary intake of purines through de novo purine nucleotide biosynthesis. UA is metabolized via various catabolic pathways and excreted via the kidney and gastrointestinal tract as well as other minor routes. ${ }^{17}$ In this study, patients with abnormal baseline sUA were excluded, and blood samples were collected after fasting 12 hours to eliminate the influence of food intake. Patients with AKI were also excluded to avoid the effects of a reduced glomerular filtration rate on UA excretion. Due to the difficulties in fecal collection and UA assessment, the excretion of UA from the gastrointestinal tract was not examined in this study. Thus, we cannot completely exclude the contribution of gastrointestinal tract excretion of UA to the change in sUA.

In this study, we found no difference between the baseline level of sUA in patients with cerebral tumors and the controls, but sUA started declining from day 1 after TBI or cerebral tumor surgery and continued to decline from day 3 until day 7. There were no detectable changes in urine UA excretion, suggesting that extra UA consumption might be one possible cause of sUA decrease.

One of the pathophysiological changes after TBI or surgery is oxidative stress. ${ }^{18}$ UA exerts nearly two-thirds of all antioxidant effects in the human body. ${ }^{19}$ Some researchers have demonstrated that UA might be consumed in the oxidative stress processes after TBI. ${ }^{7,9}$ In addition, a decrease in UA and increased end production of UA oxidation in orthotopic liver transplantation and open-heart surgery have also suggested the consumption of UA under conditions of oxidative stress. ${ }^{20,21}$ Research has also shown that extra UA could exert neuroprotective effects in animal stroke models. ${ }^{22,23}$ Recently, a study reported that oxidative stress and the inflammatory response were inhibited in a mouse model after UA treatment. ${ }^{24}$

When UA participates in antioxidant reactions, the main products are allantoin, oxonic/oxaluric and parabanic acids. ${ }^{25-28}$ Among these products, allantoin is the most stable product and is considered a good oxidative reactions biomarker in the human body. ${ }^{11}$ In this study, we examined allantoin production. Both 24-hour excretion of allantoin and allantoin/UCr were significantly decreased in patients with TBI after normalization with urine creatine to minimize renal secretion effects. There was a decrease in allantoin/sUA, suggesting that allantoin decreased faster than sUA, indicating the probability of altered UA metabolism.

In healthy organisms, norepinephrine causes a remarkable reduction in the excretion of UA via hemodynamic changes. ${ }^{29}$ In this study, we found that norepinephrine was increased in the TBI group, possibly due to trauma-induced stress. However, we did not find similar changes in patients who underwent surgery. This result suggested that norepinephrine might not be involved in the changes in serum UA.

To investigate the other possible causes of sUA changes, we assessed Pearson correlation coefficient or Spearman correlation coefficient and established a modified structural equation model. We found that plasma albumin, glucose, and calcium and phosphorus metabolism may also be associated. As a biomarker of protein nutrition status, albumin started declining on day 1 after brain injury. Previous studies have demonstrated that albumin is also a target of oxidant stress $^{19}$ and that low serum albumin may be related to poor outcomes in patients with TBI or acute spinal cord injury. ${ }^{30}$ Based on our study, decreased albumin might lead to decreased antioxidant effects and higher consumption of the antioxidant UA. Another explanation is that low albumin levels are associated with higher usage of purines to synthesize protein, resulting in low levels of UA. Higher fasting plasma glucose levels may correlate with higher levels of UA in patients with diabetes, individuals with obesity or normal individuals. ${ }^{13,31}$ However, in patients with TBI or who underwent cerebral tumor surgery, the increased Glu level was associated with a decrease in sUA, possibly due to acute stress. In our structural equation models, calcium and phosphorus metabolism were shown to have some effect on sUA changes. In addition, fewer changes in $\mathrm{Ca}^{2+}$ and Phos were found in the cerebral tumor surgery group than in the TBI group. Little is known, however, about the relationship between calcium and phosphorus metabolism, UA and brain injury. Further work is necessary to confirm the relationship and the possible causes. 
Previous studies showed there was an association between low sUA levels and worse patient outcomes. ${ }^{8}$ Other studies showed that sUA has a positive correlation with GCS. ${ }^{9}$ In this work we found that sUA levels were correlated with GCS. Hominids have evolved to generate UA as a purine metabolic end product instead of urine to obtain high brain mass as well as intellectual supremacy. ${ }^{32}$ However, clinical trials performed previously to investigate whether intravenous UA infusion can improve cognitive function in ischemic stroke patients failed to identify any significant difference with respect to mortality or long-term outcomes. ${ }^{33}$ Based on previous research, UA homeostasis and its influence on brain function, especially in pathological conditions, are worth further study.

The trend was statistically significant even though the sample size was relatively small. We do not expect increasing the sample size to alter the trend. Another limitation is the lack of direct criteria for oxidative stress, so it is difficult to rule out the influence of other antioxidant factors. Furthermore, data on UA levels in cerebrospinal fluid (CSF) are necessary to determine how UA changes in brain tissue after TBI and whether UA may pass through the blood-brain barrier to influence sUA levels. Some studies have found that UA in CSF was increased in a controlled cortical impact mouse model or released during seizure in a mouse model, ${ }^{34}$ but how UA changes in CSF remains unknown. Answering this question may help us further understand UA metabolism after TBI or surgery.

In conclusion, our study found that sUA levels decreased in patients with TBI and patients who underwent cerebral tumor surgery and were possibly related to its excess consumption as a result of its antioxidative function. Low sUA levels are associated with low GCS, suggesting an association between UA and the consciousness status of the patients. Albumin, calcium and phosphorus metabolism might be involved in UA metabolism, but further research is required to confirm this hypothesis.

Acknowledgement. We thank Dr. Li, Xingguo for his advice on this manuscript; Dr. Kang, Yi for her help on $L C-M S / M S$. We also appreciate English language editing from American Journal Experts (https://www.aje.com/).

\section{References}

1. Kanbay M, Jensen T, Solak Y, Le M, Roncal-Jimenez C, Rivard $\mathrm{C}$, et al. Uric acid in metabolic syndrome: From an innocent bystander to a central player. European Journal of Internal Medicine 2016; 29: 3-8.
2. Crotty GF, Ascherio A, Schwarzschild MA. Targeting urate to reduce oxidative stress in Parkinson disease. Experimental neurology 2017; 298: 210-224.

3. Tana C, Ticinesi A, Prati B, Nouvenne A, Meschi T. Uric Acid and Cognitive Function in Older Individuals. Nutrients 2018; 10: 975.

4. Driggin E, Helmke S, De Los Santos J, Teruya S, Guadalupe S, Goldsmith J, et al. Markers of nutritional status and inflammation in transthyretin cardiac amyloidosis: association with outcomes and the clinical phenotype. Amyloid-J Protein Fold Disord 2020; 27: 73-80.

5. Chamorro A, Dirnagl U, Urra X, Planas AM. Neuroprotection in acute stroke: Targeting excitotoxicity, oxidative and nitrosative stress, and inflammation. The Lancet Neurology 2016; 15: 869-881.

6. Vila E, Sole M, Masip N, Puertas-Umbert L, Amaro S, Dantas $\mathrm{AP}$, et al. Uric acid treatment after stroke modulates the Kruppel-like factor 2-VEGF-A axis to protect brain endothelial cell functions: Impact of hypertension. Biochem Pharmacol 2019; 164: 115-128.

7. Llull L, Renu A, Laredo C, Codas J, Rudilosso S, Urra X, et al. Uric acid in subarachnoid hemorrhage patients: Temporal profile and its association to neurological complications. International Journal of Stroke 2015; 10: 292.

8. Hatefi M, Dastjerdi MM, Ghiasi B, Rahmani A. Association of serum uric acid level with the severity of brain injury and patient's outcome in severe traumatic brain injury. Journal of Clinical and Diagnostic Research 2016; 10: OC20-OC4.

9. Adiga US, Vickneshwaran V, Sen SK, Thiagaragan G. Bilirubin and uric acid in traumatic brain injury. Journal of Global Pharma Technology 2011; 3: 20-24.

10. Lu Z, Dong B, Wu H, Chen T, Zhang Y, Wu J, et al. Serum uric acid level in primary hypertension among Chinese nonagenarians/centenarians. J Hum Hypertens 2009; 23: 113-121.

11. Martinez-Moral MP, Kannan K. Allantoin as a Marker of Oxidative Stress: Inter- and Intraindividual Variability in Urinary Concentrations in Healthy Individuals. Environmental Science \& Technology Letters 2019; 6: 283-288.

12. Keenan RT. The biology of urate. Seminars in Arthritis and Rheumatism 2020; 50: S2-S10.

13. Caliceti C, Calabria D, Roda A, Cicero AFG. Fructose Intake, Serum Uric Acid, and Cardiometabolic Disorders: A Critical Review. Nutrients 2017; 9: 15.

14. Virdis A, Masi S, Casiglia E, Tikhonoff V, Cicero AFG, Ungar A, et al. Identification of the Uric Acid Thresholds Predicting an Increased Total and Cardiovascular Mortality Over 20 Years. Hypertension 2020; 75: 302-308.

15. Li JJ, Huang YH, Lin YY, Li MM, Chen YF, Cai RW. The association of uric acid with leukoaraiosis. Journal of International Medical Research 2017; 45: 75-81.

16. Spiga R, Marini MA, Mancuso E, Di Fatta C, Fuoco A, Perticone F, et al. Uric Acid Is Associated With Inflammatory Biomarkers and Induces Inflammation Via Activating the NF-kappaB Signaling Pathway in HepG2 Cells. Arteriosclerosis, Thrombosis \& Vascular Biology 2017; 37: 1241-1249.

17. Maiuolo J, Oppedisano F, Gratteri S, Muscoli C, Mollace V. Regulation of uric acid metabolism and excretion. Int J Cardiol 2016; 213: 8-14.

18. Di Pietro V, Yakoub KM, Caruso G, Lazzarino G, Signoretti $\mathrm{S}$, Barbey AK, et al. Antioxidant Therapies in Traumatic Brain Injury. Antioxidants 2020; 9: 34. 
19. Mironczuk-Chodakowska I, Witkowska AM, Zujko ME. Endogenous non-enzymatic antioxidants in the human body. Advances in Medical Sciences 2018; 63: 68-78.

20. Lu HY, Ning XY, Chen YQ, Han SJ, Chi P, Zhu SN, et al. Predictive Value of Serum Creatinine, Blood Urea Nitrogen, Uric Acid, and beta2-Microglobulin in the Evaluation of Acute Kidney Injury after Orthotopic Liver Transplantation. Chin Med J (Engl) 2018; 131: 1059-1066.

21. Gaipov A, Solak Y, Turkmen K, Toker A, Baysal AN, Cicekler $\mathrm{H}$, et al. Serum uric acid may predict development of progressive acute kidney injury after open heart surgery. Ren Fail 2015; 37: 96-102.

22. Aliena-Valero A, Lopez-Morales MA, Burguete M, CastelloRuiz M, Jover-Mengual T, Hervas D, et al. Emergent Uric Acid Treatment is Synergistic with Mechanical Recanalization in Improving Stroke Outcomes in Male and Female Rats. Neuroscience 2018; 388: 263-273.

23. Cutler RG, Camandola S, Feldman NH, Yoon JS, Haran JB, Arguelles S, et al. Uric acid enhances longevity and endurance and protects the brain against ischemia. Neurobiology of Aging 2019; 75: 159-168.

24. Liu H, He J, Zhong J, Zhang H, Zhang Z, Liu L, et al. Clinical and Basic Evaluation of the Prognostic Value of Uric Acid in Traumatic Brain Injury. Int J Med Sci 2018; 15: 1072-1082.

25. Iida S, Yamamoto Y, Susa C, Tsukui K, Fujisawa A. 5-N-Carboxyimino-6-N-chloroaminopyrimidine-2,4(3H)dione as a hypochlorite-specific oxidation product of uric acid. Journal of Clinical Biochemistry and Nutrition 2018; 63: 85-89.

26. Lida S, Ohkubo Y, Yamamoto Y, Fujisawa A. Parabanic acid is the singlet oxygen specific oxidation product of uric acid. Journal of Clinical Biochemistry and Nutrition 2017; 61: 169-175.

27. Kozlik P, Hasikova L, Stiburkova B, Zavada J, Kalikova K. Rapid and reliable HILIC-MS/MS method for monitoring allantoin as a biomarker of oxidative stress. Analytical Biochemistry 2020; 589: 5.
28. Esiaba I, Angeles DM, Holden MS, Tan JBC, Asmerom Y, Gollin G, et al. Urinary Allantoin Is Elevated in Severe Intraventricular Hemorrhage in the Preterm Newborn. Translational Stroke Research 2016; 7: 97-102.

29. Mandal AK, Mount DB. The Molecular Physiology of Uric Acid Homeostasis. In: Julius D, editor. Annual Review of Physiology, Vol 77. Annual Review of Physiology. 77. Palo Alto: Annual Reviews; 2015. p. 323-45.

30. Tong BB, Jutzeler CR, Cragg JJ, Grassner L, Schwab JM, Casha $S$, et al. Serum Albumin Predicts Long-Term Neurological Outcomes After Acute Spinal Cord Injury. Neurorehabilitation and Neural Repair 2018; 32: 7-17.

31. Cicero AF, Rosticci M, Bove M, Fogacci F, Giovannini M, Urso R, et al. Serum uric acid change and modification of blood pressure and fasting plasma glucose in an overall healthy population sample: data from the Brisighella heart study. Ann Med 2017; 49: 275-282.

32. De Giorgi A, Fabbian F, Pala M, Tiseo R, Parisi C, Misurati E, et al. Uric acid: friend or foe? Uric acid and cognitive function "Gout kills more wise men than simple". Eur Rev Med Pharmacol Sci 2015; 19: 640-646.

33. Amaro S, Renu A, Laredo C, Castellanos M, Arenillas JF, Llull L, et al. Relevance of Collaterals for the Success of Neuroprotective Therapies in Acute Ischemic Stroke: Insights from the Randomized URICO-ICTUS Trial. Cerebrovascular Diseases 2019; 47: 171-177.

34. Thyrion L, Raedt R, Portelli J, Van Loo P, Wadman WJ, Glorieux $G$, et al. Uric acid is released in the brain during seizure activity and increases severity of seizures in a mouse model for acute limbic seizures. Experimental neurology 2016; 277: 244-251. 